\title{
EFIE and MFIE, Why the Difference?
}

\author{
W. C. Chew*1, C. P. Davis ${ }^{2}$, K. F. Warnick ${ }^{3}$, Z. P. Nie ${ }^{4}$, J. Hu ${ }^{4}$, S. Yan ${ }^{4}$, and \\ L. Gürel ${ }^{5}$ \\ ${ }^{1}$ The University of Hong Kong \\ (On leave of absence from the University of Illinois at Urbana-Champaign), \\ email:w-chew@uiuc.edu \\ ${ }^{2}$ University of Illinois at Urbana-Champaign, USA, email : \\ claytonpdavis@gmail.com \\ ${ }^{3}$ Brigham Young University, USA, email: warnick@ee.byu.edu \\ ${ }^{4}$ University of Electronic Science and Technology of China, email: \\ zpnie@uestc.edu.cn \\ ${ }^{5}$ Bilkent University, Turkey, email: lgurel@bilkent.edu.tr
}

\begin{abstract}
EFIE (electric field integral equation) suffers from internal resonance, and the remedy is to use MFIE (magnetic field integral equation) to come up with a CFIE (combined field integral equation) to remove the internal resonance problem. However, MFIE is fundamentally a very different integral equation from EFIE. Many questions have been raised about the differences.
\end{abstract}

First, it has often been observed that EFIE has better accuracy than MFIE. On the other hand, MFIE has better convergence rate when solved with an iterative solver [1,2]. Also, EFIE has low-frequency breakdown, but MFIE does not have an apparent low-frequency problem [3].

We will perform error analysis to explain why EFIE has better accuracy compared to MFIE [4-7]. Mathematical analysis shows that EFIE has a smoothing operator, while MFIE has a non-smoothing operator [8-10]. This difference often gives rise to better accuracy for EFIE compared to MFIE.

MFIE is a second kind integral equation while EFIE is a first kind integral equation [10]. Hence, the eigenvalues of the EFIE operator tends to cluster around the origin, while the eigenvalues of the MFIE operator are shifted away from the origin. Consequently, when solved with an iterative solver, the convergence behavior of MFIE is superior to that of EFIE.

It is well-known that EFIE suffers from the low-frequency breakdown problem. MFIE does not suffer from apparent low-frequency breakdown, but it suffers from low-frequency inaccuracy [3]. All these problems can be taken care of by performing the loop-tree decomposition.

The EFIE operator is often known as the $\mathcal{L}$ operator and the MFIE operator is often known as the $\mathcal{K}$ operator in the literature. The $\mathcal{L}$ operator is a symmetric operator while the $\mathcal{K}$ operator is an asymmetric operator. In some integral equations such as those involving dielectric interfaces, these two operators appear simultaneously. They also appear concurrently in the invocation of the equivalence principle. Their discretization often gives rise 
to an ill-conditioned matrix representation. We will discuss the reasons and present some remedies for them. More will be discussed at the conference presentation.

\section{References}

[1] C. P. Davis and K. F. Warnick, "Error analysis of 2D MoM for MFIE/EFIE/CFIE based on the circular cylinder, IEEE Trans. Ant. Propag., vol. 53, pp. 321331, January 2005 .

[2] Ö. Ergül and L. Gürel, "Improved testing of the magnetic-field integral equation, IEEE Antennas Wireless Propag. Lett., vol. 15, pp. 615-617, 2005.

[3] Y. Zhang, T. J. Cui, W. C. Chew, and J. S. Zhao, "Magnetic field integral equation at very low frequencies," IEEE Trans. Antennas Propagat., vol. 51, no. 8, pp. 18641871, Aug 2003.

[4] D. G. Dudley, "Error minimization and covergence in numerical methods," Electromagnetics, , vol. 5, pp. 8997, 1985.

[5] A. F. Peterson, D. R. Wilton, and R. E. Jorgenson, IEEE Trans. Antennas Propagat., vol. 27, pp. 241242, Apr. 1996.

[6] D. G. Dudley, "Comments on Variational nature of Galerkin and non-Galerkin moment method solutions, IEEE Trans. Ant. Propag., vol. 45, June 1997.

[7] K. F. Warnick and W. C. Chew, "Accuracy of the method of moments for scattering by a cylinder, IEEE Trans. Micr. Th. Tech., vol. 48, pp. 16521660, Oct. 2000.

[8] E. F. Kuester, "Computable error bounds for variational functionals of solutions of a convolution integral equations of the first kind", Wave Motion, vol. 22, pp. 171-185, 1995.

[9] S. Amini and S. M. Kirkup, "Solution of Helmholtz equation in the exterior domain by elementary boundary integral methods", J. Comp. Phys., vol. 118, pp. 208-221, 1995.

[10] G. C. Hsiao and R. E. Kleinman, "Mathematical foundations for error estimation in numerical solutions of integral equations in electromagnetics," IEEE Trans. Ant. Propag., vol. 45, no. 3, pp. 316-328,1997. 